ARTICLE

DOI: $10.1038 / s 41467-017-02698-4$

\title{
Dual catalysis for enantioselective convergent synthesis of enantiopure vicinal amino alcohols
}

\author{
Chen-Xi Ye (1) ${ }^{1}$, Yared Yohannes Melcamu ${ }^{1}{ }^{12,3}$, Heng-Hui Li ${ }^{1}$, Jiang-Tao Cheng ${ }^{1}$, Tian-Tian Zhang ${ }^{1}$, \\ Yuan-Ping Ruan ${ }^{1}$, Xiao Zheng ${ }^{1,4}$, Xin Lu ${ }^{1,2,3}$ \& Pei-Qiang Huang (1D ${ }^{1,2}$
}

Enantiopure vicinal amino alcohols and derivatives are essential structural motifs in natural products and pharmaceutically active molecules, and serve as main chiral sources in asymmetric synthesis. Currently known asymmetric catalytic protocols for this class of compounds are still rare and often suffer from limited scope of substrates, relatively low regio- or stereoselectivities, thus prompting the development of more effective methodologies. Herein we report a dual catalytic strategy for the convergent enantioselective synthesis of vicinal amino alcohols. The method features a radical-type Zimmerman-Traxler transition state formed from a rare earth metal with a nitrone and an aromatic ketyl radical in the presence of chiral $N_{1} N^{\prime}$-dioxide ligands. In addition to high level of enantio- and diastereoselectivities, our synthetic protocol affords advantages of simple operation, mild conditions, high-yielding, and a broad scope of substrates. Furthermore, this protocol has been successfully applied to the concise synthesis of pharmaceutically valuable compounds (e.g., ephedrine and selegiline).

\footnotetext{
${ }^{1}$ Department of Chemistry and Fujian Provincial Key Laboratory of Chemical Biology, College of Chemistry and Chemical Engineering, Xiamen University, Xiamen, Fujian 361005, China. ${ }^{2}$ Collaborative Innovation Center of Chemistry for Energy Materials, Xiamen University, Xiamen, Fujian 361005 , China. ${ }^{3}$ State Key Laboratory of Physical Chemistry of Solid Surfaces, Xiamen University, Xiamen, Fujian 3 361005, China. ${ }^{4}$ Key Laboratory of Synthetic Chemistry of Natural Substances, Shanghai Institute of Organic Chemistry, Chinese Academy of Sciences, Shanghai 200032, China. Correspondence and requests for materials should be addressed to X.Z. (email: zxiao@xmu.edu.cn) or to X.L. (email: xinlu@xmu.edu.cn) or to P.-Q.H. (email: pqhuang@xmu.edu.cn)
} 
E nantiopure vicinal amino alcohols and their derivatives represent one of the most significant synthetic building blocks and key subunits of pharmaceutically active molecules, chiral auxiliaries and ligands. Synthesis of such compounds has stimulated continuing interest and extensive efforts ${ }^{1-9}$. Traditional methods for this aim are such addition reactions that mostly require enantiopure substrates or reagents, including functional group transformation of vicinal $\mathrm{N}, \mathrm{O}$-compounds ${ }^{1-3}$, addition of $N / O$-heteroatoms to substrates ${ }^{4-7}$, and nitro-group's derivatization via nucleophilic nitroaldol (Henry) reaction ${ }^{8}$ (I-III in Fig. 1a). However, these strategies, including their catalytic enantioselective evolutions ${ }^{3,6-8}$, suffered from either structurally limited substrates/products or relatively low regioselectivity.

Compared with the above protocols, radical cross-coupling between amine and alcohol moieties represents an inherently efficient and flexible way for construction of vicinal amino alcohols (Fig. 1a-IV). By using $\mathrm{SmI}_{2}$ as reductant and oxophilic coordination center, reductive cross-coupling of imine derivatives ${ }^{9-11}$ or nitrones ${ }^{12-15}$ with carbonyl compounds (e.g., aldehydes/ketones) allows for an easy access to these compounds with various structures. However, the use of $\mathrm{SmI}_{2}$ in stoichiometric quantity poses a substantial challenge for enantioselective induction from chiral ligands ${ }^{16}$, along with unavoidable side reactions such as pinacoltype homocoupling and reduction of substrates (Fig. 1b). Recently, photocatalysis $^{17-22}$ also provided several schemes on vicinal amino alcohols and their derivatives ${ }^{23-28}$, including three enantioselective protocols catalyzed by photocatalyst-merged dual catalyst systems with chiral phosphoric acid organocatalyst ${ }^{23}$ or chiral rhodium Lewis acid ${ }^{24}$, as well as bifunctional Lewis acid/photoredox catalys $^{25}$ of chiral-at-metal iridium complex ${ }^{29}$. Nevertheless, all of these methods relied heavily on specially designed substrates.

We recently envisioned that an efficient and flexible strategy for enantiopure vicinal amino alcohols might be realized by aptly combining merits of the aforementioned $\mathrm{SmI}_{2}$-mediated ${ }^{12-15}$ and photocatalytic ${ }^{23-28}$ protocols from nitrones and aldehydes. That is, a photocatalytic protocol featuring with intermolecular singleelectron-transfer (SET) can be used to reduce selectively the substrate of higher electron affinity (i.e., higher reduction potential ${ }^{26,30}$ and an oxophilic Lewis acid co-catalyst (e.g., rare earth metal cation) can be introduced to bind simultaneously both substrates ${ }^{31,32}$, and, more importantly, an appropriate chiral ligand to form precursor complex I and to induce desired enantioselectivity likely through a radical-type
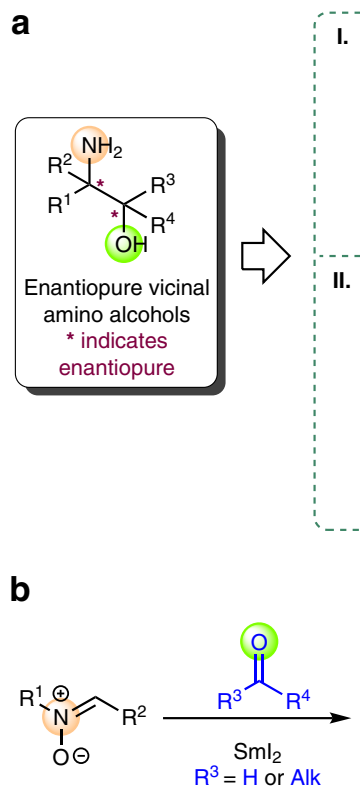
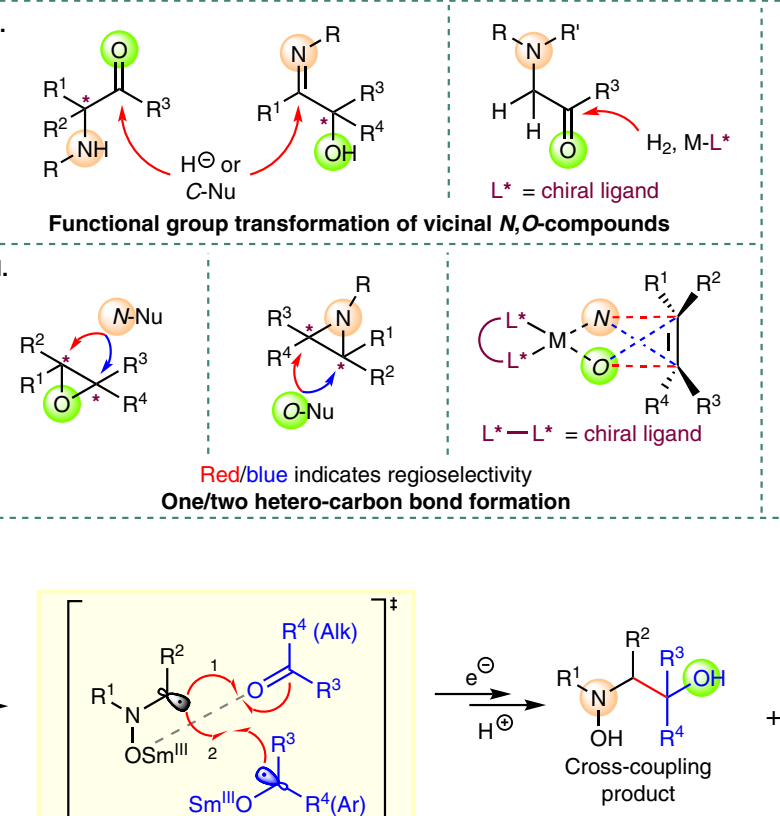

Functional group transformation of vicinal $N, O$-compounds

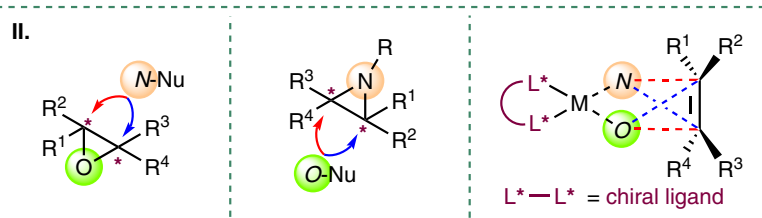

Red/blue indicates regioselectivity

One/two hetero-carbon bond formation

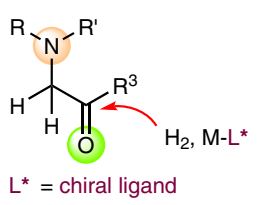

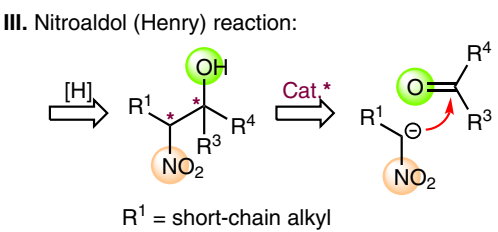

IV. Radical coupling strategy:

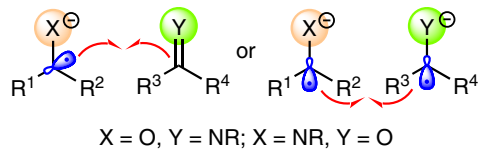

Promoted by: a) $\mathrm{Sml}_{2}$; b) photocatalysis Convergent carbon-carbon bond formation

C
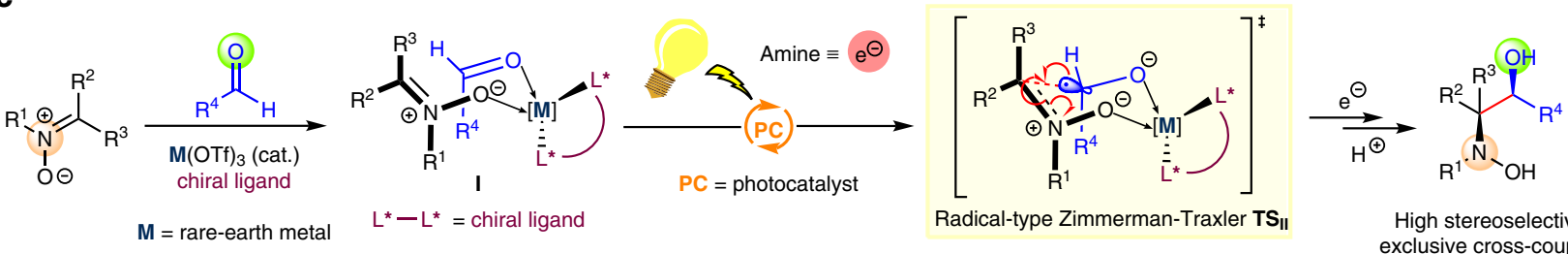

High stereoselectivity exclusive cross-coupling

Fig. 1 Retrosynthetic analysis and synthetic protocols of enantiopure vicinal amino alcohols. a The general protocols for synthesis of enantiopure vicinal amino alcohols and their derivatives. b Previous studies by Py and our group on $\mathrm{Sml}_{2}$-mediated cross-coupling of nitrones with aldehydes/ketones may lead to homocoupling and reduction byproducts and are not ideally suitable for developing catalytic enantioselective variant. c Enantioselective reductive cross-coupling reaction of nitrones with aromatic aldehydes via the synergistic catalysis of chiral ligand-coordinated Lewis acid and photocatalyst was described. Through the radical-type Zimmerman-Traxler $\mathbf{T S}_{\mathbf{I I}}$, vicinal hydroxyamino alcohols could be obtained exclusively with high stereoselectivity. This mild reaction is operationally simple with a wide array of nitrones and aromatic aldehydes 


\section{Table 1 Photocatalytic enantioselective synthesis of vicinal hydroxyamino alcohol $2 a-1$}

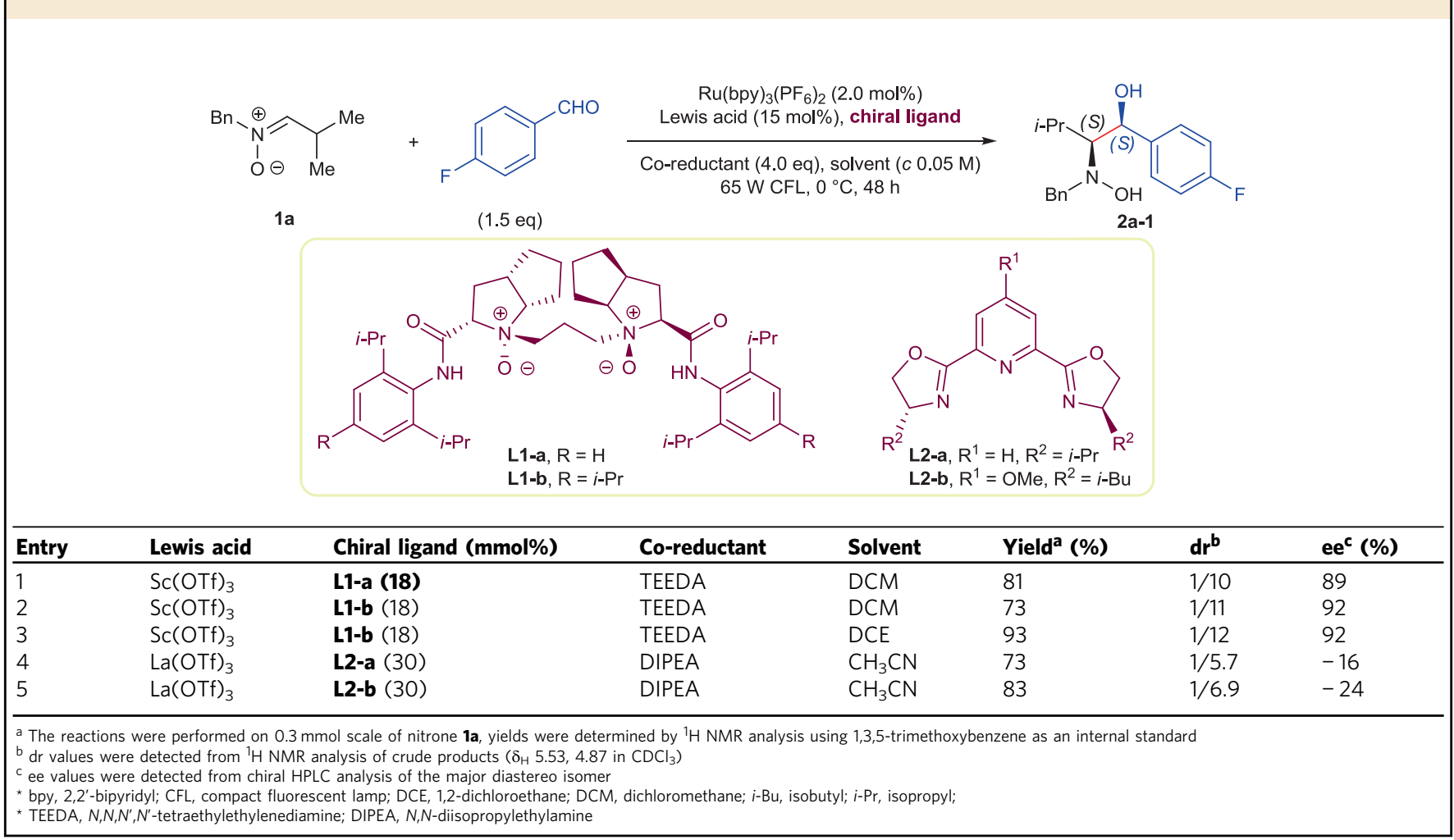

Zimmerman-Traxler transition state $\mathbf{T S}_{\mathbf{I I}}{ }^{33,34}$ in the subsequent cross-coupling. Herein we report a synergistic catalysis of chiral $N, N^{\prime}$-dioxides (Feng's ligands) ${ }^{35,36}$ coordinated rare earth ion and Ru-photocatalyst for enantioselective radical convergent synthesis of enantiopure vicinal hydroxyamino alcohols from nitrones and aromatic aldehydes, together with a mechanism deciphering catalytic cycle and stereoselectivity of this reaction.

\section{Results}

Optimization of the reaction conditions. Our investigation into this dual catalysis protocol began by studying the model reaction of nitrone 1a with 4-fluorobenzaldehyde under various conditions (Table 1). Initial experiments revealed the synergistic catalytic effect of Lewis acid and photocatalyst, which led to desired vicinal hydroxyamino alcohol $( \pm)-\mathbf{2 a - 1}$. Among several chiral ligand classes examined (see Supplementary Table 1), Feng's chiral $N, N^{\prime}$-dioxides (such as $\mathbf{L 1 - a}$ and L1-b), which are wellknown privileged chiral catalysts ${ }^{37}$, provided enantioselective results for this reductive coupling reaction (see Supplementary Tables 2 and 3), despite that this class of ligands has not proved to be effective for asymmetric radical reaction before ${ }^{35,36}$. Meanwhile, we noted that chiral $N, N^{\prime}$-dioxides were potentially reduced by this dual-catalyst system owing to the oxidation ability of tertiary amine oxides (see Supplementary Note 1 for details). Fortunately, by optimization of Lewis acids we found that rare earth ions coordinated $N, N^{\prime}$-dioxides were stable enough under our conditions to give optically enriched product. PyBOX ligands, which are originally utilized by Yoon and colleagues ${ }^{38}$ for photocatalyst/chiral Lewis acid dual-activation catalysis, also provided enantioselectivity but only with low ee (such as L2-a and L2-b). Other optimization studies involving chiral ligands, Lewis acids, co-reductants, temperature and solvents were performed.
Finally, by using a $65 \mathrm{~W}$ compact fluorescent lamp (CFL) as the light source, the coupling of nitrone 1 a with 4fluorobenzaldehyde was carried out in the presence of $\mathrm{Ru}$ (bpy) $)_{3}\left(\mathrm{PF}_{6}\right)_{2}(2.0 \mathrm{~mol} \%), \mathrm{Sc}(\mathrm{OTf})_{3}(15 \mathrm{~mol} \%), \mathbf{L 1}-\mathbf{b}(18 \mathrm{~mol} \%)$, and $N, N, N^{\prime}, N^{\prime}$-tetraethylethylenediamine (TEEDA, 4.0 eq) in 1,2dichloroethane (DCE) at $0{ }^{\circ} \mathrm{C}$ for $48 \mathrm{~h}$ to offer the desired product 2a-1 in good yield ( $93 \%$ combined yield) with high diastereoselectivity and enantioselectivity (1/12 dr and $92 \%$ ee).

Enantioselective reductive cross-coupling of nitrones with aldehydes. With these optimized conditions in hand, we next examined the scope of nitrones. As illustrated in Table 2, symmetrical ketonitrones $\mathbf{1 b}$ to $\mathbf{1 d}$ gave the desired vicinal hydroxyamino alcohols with single chiral centers, respectively, in moderate to good yields with excellent enantioselectivity (Table 2, entries 2-6). The absolute configuration of $\mathbf{2 b} \mathbf{b} \mathbf{3}$ was determined to be $(S)$ by derivatization ${ }^{39}$ and single-crystal X-ray diffraction analysis (see Supplementary Fig. 2). Notably, as for these symmetrical ketonitrones, dichloromethane (DCM) was better than DCE. Next, we turned our attention to the scope of aldonitrones. Compared with nitrone 1a, more or less steric hindrance of substituents $R^{3}\left(R^{2}=\mathrm{H}\right)$ of nitrones diminished the reaction yields and stereoselectivity (Table 2, entry 1 vs. entries $7-10$ ). The influences of $\mathrm{N}$-alkyl substituent groups of nitrones were also investigated and $N$-benzyl nitrone la provided a better result than $N$-methyl nitrone $\mathbf{1 i}$ and $N$-isopropyl nitrone $\mathbf{1 j}$ (Table 2, entry 1 vs. entries 11 and 12).

We also explored the scope of aldehydes and found aromatic aldehydes to be excellent partners with nitrone 1a; aliphatic aldehydes, which were compatible with previous $\mathrm{SmI}_{2}$-mediated conditions $^{12}$, are unavailable for this reaction. As for parasubstituted aromatic aldehydes, the more electron-deficient one 


\section{Table 2 Enantioselective reductive cross-coupling of nitrones with aldehydes ${ }^{a}$}
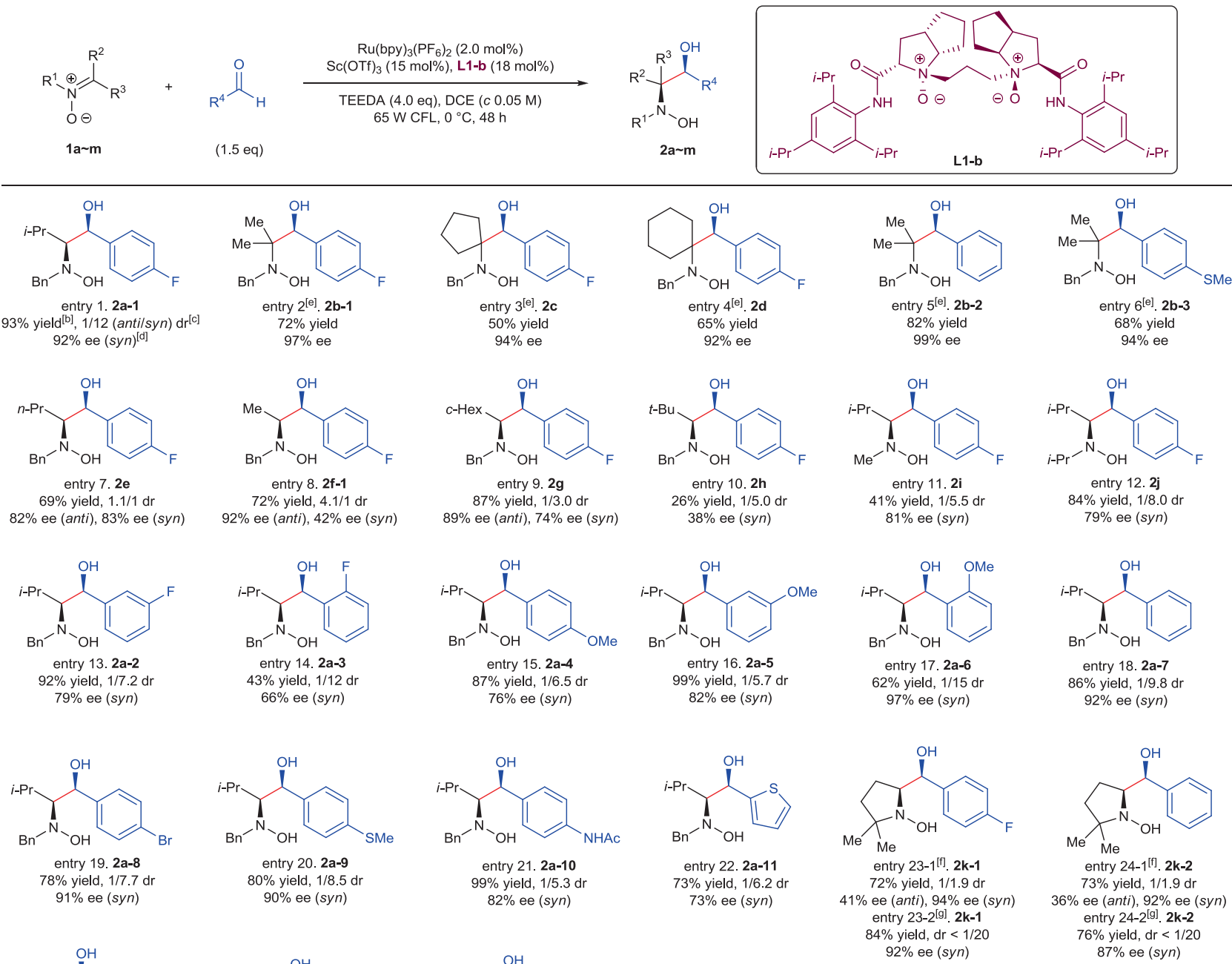<smiles>COc1ccc(C(O)C2CCC(C)(C)N2O)cc1</smiles>

$\mathrm{Me}{ }_{\mathrm{M}}$

entry $25-1^{[\mathrm{ff}} . \mathbf{2 k - 3}$

$94 \%$ yield, $1 / 2.0 \mathrm{dr}$

$49 \%$ ee (anti), $95 \%$ ee (syn)

entry $25-2^{[\mathrm{g}]}$. 2k-3

$94 \%$ yield, $d r<1 / 20$

$89 \%$ ee $(s y n)$

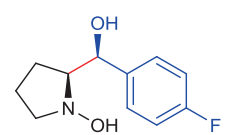

entry $26-1^{[\mathrm{fl}]} .21$ $72 \%$ yield, $1 / 2.1 \mathrm{dr}$

$4 \%$ ee (anti), $97 \%$ ee (syn)

entry $26-2^{[\mathrm{g}]}$. 21

$64 \%$ yield, $1 / 12 \mathrm{dr}$

$72 \%$ ee (syn)

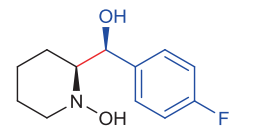

entry $27-1^{[\text {[f] }} .2 \mathrm{~m}$

$58 \%$ yield, $1 / 9.9 \mathrm{dr}$

$97 \%$ ee (syn)

entry $27-2^{[\mathrm{g}]} .2 \mathrm{~m}$

$47 \%$ yield, $1 / 20 \mathrm{~d}$
$57 \%$ ee $($ syn $)$

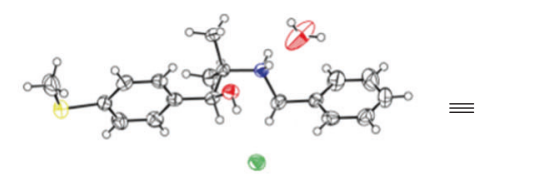

X-ray of $6 \mathrm{~b}-3 \cdot \mathrm{HCl} \cdot \mathrm{H}_{2} \mathrm{O}$

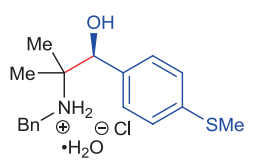

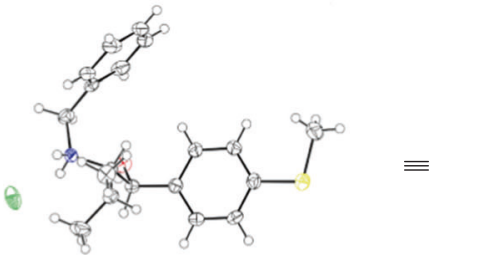

X-ray of $6 \mathrm{a}-9 \cdot \mathrm{HCl}$

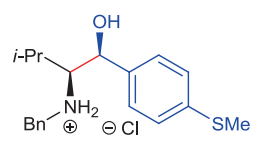

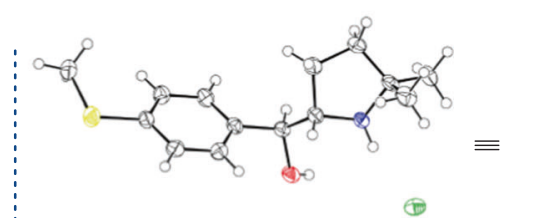

$\Phi$

X-ray of $6 \mathrm{k}-3 \cdot \mathrm{HCl}$

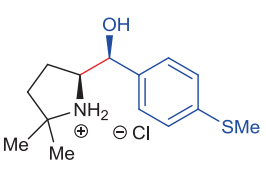

${ }^{a}$ General method: Ru(bpy) ${ }_{3}\left(\mathrm{PF}_{6}\right)_{2}(2.0 \mathrm{~mol} \%), \mathrm{Sc}(\mathrm{OTf})_{3}(15 \mathrm{~mol} \%)$, L1-b $(18 \mathrm{~mol} \%), \mathrm{DCE}(\mathrm{c} 0.05 \mathrm{M}), 65 \mathrm{~W} \mathrm{CFL}, 0{ }^{\circ} \mathrm{C}, 48 \mathrm{~h}$

b Isolated yield

${ }^{c} \mathrm{dr}$ values (anti/syn) were detected from ${ }^{1} \mathrm{H}$ NMR or chiral HPLC analysis of crude products

${ }^{d}$ ee values were detected from chiral HPLC analysis

DCM was used as solvent

${ }^{\mathrm{f}}$ Modified method 1: Ru(bpy) ${ }_{3}\left(\mathrm{PF}_{6}\right)_{2}(2.0 \mathrm{~mol} \%), \mathrm{Sc}(\mathrm{OTf})_{3}(15 \mathrm{~mol} \%)$, L1-a $(18 \mathrm{~mol} \%)$, DIPEA (4.0 eq), DCM (c $\left.0.05 \mathrm{M}\right), 65 \mathrm{~W} \mathrm{CFL},-5^{\circ} \mathrm{C}, 48 \mathrm{~h}$

${ }^{g}$ Modified method 2: $\mathrm{Ru}(\mathrm{bpy})_{3}\left(\mathrm{PF}_{6}\right)_{2}(2.0 \mathrm{~mol} \%), \mathrm{La}(\mathrm{OTf})_{3}(15 \mathrm{~mol} \%), \mathbf{L 2}-\mathbf{b}(30 \mathrm{~mol} \%)$, TEEDA $(4.0 \mathrm{eq}), \mathrm{CH}_{3} \mathrm{CN}(\mathrm{c} 0.05 \mathrm{M}), 65 \mathrm{~W} \mathrm{CFL},-10^{\circ} \mathrm{C}, 72 \mathrm{~h}$ 


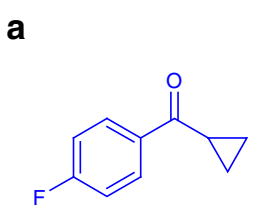

3 (1.0 eq)
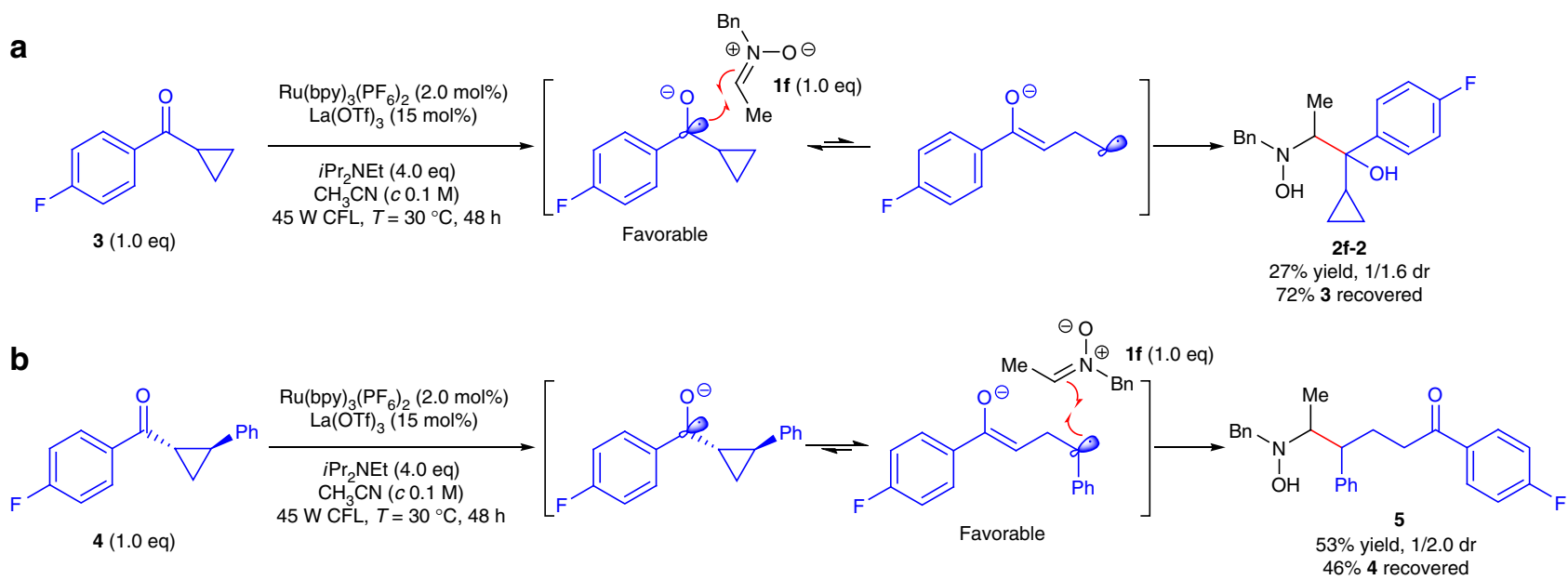

Fig. 2 Radical clock reactions. a The normal cross-coupling was observed without ring opening product from ketone $\mathbf{3}$. b The radical clock generated from well-designed radical clock precursor $\mathbf{4}$ was rearranged and added to nitrone $1 \mathbf{f}$

gave the higher stereoselectivity (Table 2, entries $1,15,18$ to 21 ), whereas ortho- and meta-substituted aromatic aldehydes were opposite to this (Table 2, entry 14 vs. entry 17, entry 13 vs. entry 16). Moreover, a series of functional groups including thioether, secondary amide and thiophene were well tolerated in this reaction (Table 2, entries 20-22).

Furthermore, by using L1-a as chiral ligand and $N, N-$ diisopropylethylamine (DIPEA) instead of TEEDA (see Methods, modified method 1), cyclic nitrone ( $2 \mathbf{k}$ to $\mathbf{2 m}$ ) also can be crosscoupled with aromatic aldehydes in moderate to excellent yields with high enantioselectivity but low diastereoselectivity (Table 2, entries $23-27 ; 58-94 \%$ combined yields, $92-97 \%$ ee, $1 / 1.9-1 / 9.9$ $\mathrm{dr})$. It is noteworthy that, by using a complex of $\mathrm{La}(\mathrm{OTf})_{3}$ with PyBOX ligand L2-b as the chiral Lewis acid (see Methods, modified method 2), reductive cross-coupling of nitrone $\mathbf{1 k}$ with aromatic aldehydes can also offer the desired products in goodto-excellent yields with high stereoselectivity (Table 2, entries 23-25; 76-94\% combined yield, $87-92 \%$ ee, dr <1/20). Nevertheless, this modified condition was not quite compatible with nitrones $\mathbf{1 l}$ and $\mathbf{1 m}$. The absolute configuration of vicinal hydroxyamino alcohols $\mathbf{2 a - 9}$ and $\mathbf{2 k - 3}$ were both determined to be $(S, S)$ by single-crystal X-ray diffraction analysis (see Supplementary Fig. 2). Notably, aromatic ketones can also cross-couple with nitrones smoothly under racemic photocatalytic conditions to produce the desired vicinal hydroxyamino alcohols. However, following the general method or modified methods mentioned in Table 2, only traces of desired products were observed. In addition, aromatic nitrones, such as $\mathrm{N}$-benzylbenzylidene amine oxide (1o, see Supplementary Fig. 3), always provided complex results in this reaction.

Mechanistic investigations. A series of experiments were carried out to get a deep insight into this reaction (see Supplementary Discussion). Control experiments showed no product was formed in the absence of photocatalyst, Lewis acid, amine reductant or light source, thus established that the reaction is synergistically catalyzed by Lewis acid and photocatalyst through a light-driven reductive process. Moreover, a radical mechanism is consistent with the phenomenon that photoreaction was entirely inhibited when 1 equivalent TEMPO (2,2,6,6-tetramethylpiperidine-1-oxyl) was added to the reaction mixture. We postulated that this photocatalytic reaction, unlike the previous $\mathrm{SmI}_{2}$-mediated reactions, is initiated by the visible light excited SET reduction of aldehydes to ketyl radicals. To verify our hypothesis, radical clock reactions of nitrone If under the racemic photocatalytic condition were carried out (Fig. 2). Considering the ring opening of $\alpha$ cyclopropylbenzyl radical is not a thermodynamic feasible process $^{40}$ (e.g., with 4-fluorophenyl ketone 3, vicinal hydroxyamino alcohol $\mathbf{2 f - 2}$ was obtained in $27 \%$ yield as a $1 / 1.6$ (minor/major) mixture of inseparable diastereomers, along with $72 \%$ of recovered ketone 3), we designed and synthesized cyclopropylcontaining ketone 4 as a radical clock precursor ${ }^{41}$. Cyclopropyl opening of ketone 4 followed by cross-coupling with nitrone 1f provided $\delta$-hydroxyamino ketone $\mathbf{5}$ in $53 \%$ yield as a $1 / 2.0$ (minor/major) mixture of diastereomers, along with $46 \%$ of recovered ketone 4 . Thus, the radical clock reactions proved that our photocatalytic reaction of nitrones with aldehydes is initiated by the SET reduction of aldehydes.

The hypothesis was supported by the cyclic voltammetry studies and density functional theory (DFT) calculations (see Supplementary Fig. $4 \sim 6$ and $8 \sim 11$ ). The coordination of 4-fluorobenzaldehyde with $\mathrm{Sc}(\mathrm{OTf})_{3}$ in $\mathrm{CH}_{3} \mathrm{CN}$ resulted in a significant reduction in the aldehyde's half-wave potential which shifted from $-1.86 \mathrm{~V}$ to $-0.62 \mathrm{~V}$ [vs. saturated calomel electrode (SCE)]. With the fact that nitrone has a higher affinity with Lewis acid (see Supplementary Fig. 7, the reactants' solubility studies), a complex A of nitrone, aldehyde and Lewis acid was supposed to be a plausible starting point of the reaction, which leads to the proposed mechanism shown in Fig. 3. Photoexcitation and reductive quenching of $\mathrm{Ru}(\mathrm{bpy})_{3}{ }^{2+}$ by DIPEA affords $\left[i \mathrm{Pr}_{2}(\mathrm{Et}) \mathrm{N} \cdot\right]$ ${ }^{+}$and $\mathrm{Ru}(\mathrm{bpy})_{3}{ }^{+}\left(E_{1 / 2}{ }^{\mathrm{II} / \mathrm{I}}=-1.33 \mathrm{~V}\right.$ vs. SCE in $\left.\mathrm{MeCN}^{42,43}\right)$, which is sufficient to reduce complex $\mathbf{A}$ by intermolecular SET (onset potential $E_{\mathrm{op}}>-0.5 \mathrm{~V}$ vs. SCE) and generate the radical complex B. Indeed, DFT calculations confirmed that the electron affinity of $\mathbf{A}$ is much higher ( $~ 63.0 \mathrm{kcal} \mathrm{mol}^{-1}$ in free energy) than that of nitrone 1a $\left(\sim 23.4 \mathrm{kcal} \mathrm{mol}^{-1}\right)$ and 4-fluorobenzaldehyde $(\sim 45.1$ $\mathrm{kcal} \mathrm{mol}^{-1}$ ) in solvent, and the as-generated cross-coupling precursor $\mathbf{B}$ has spin density localized predominantly on the aldehyde moiety. Subsequently, $\mathrm{N}$-radical intermediate $\mathbf{C}$ (or $\mathbf{C}^{\prime}$ of anti-configuration) is formed through an analogous 6-endotrig radical annulation and the transition state $\mathbf{T S}_{\mathbf{B}}$ leading to a syn-configuration is predicted to be by $1.9 \mathrm{kcal} \mathrm{mol}^{-1}$ favored over the anti-configuration transition state $\mathbf{T S}_{\mathbf{B}^{\prime}}$. $\mathbf{C}$ upon hydrogen abstraction from $\left[i \mathrm{Pr}_{2}(\mathrm{Et}) \mathrm{N} \cdot\right]^{+}$affords regioselectively intermediate $\mathbf{D}$ (via $\mathbf{T S}_{\mathbf{C}}$ ) other than $\mathbf{D}^{\prime}$ (via $\mathbf{T S}_{\mathbf{C}^{\prime}}$ ). Finally, protonation of $\mathbf{D}$ gives the desired vicinal hydroxyamino alcohol 2a-1 as a major diastereomer. Moreover, DFT calculations also showed that the formation of cross-coupling precursor $\mathbf{B}$ is overwhelmingly favored over the formation of homocoupling 
Model reaction:<smiles>CC(C)/C=[N+](\[O-])Cc1ccccc1</smiles>

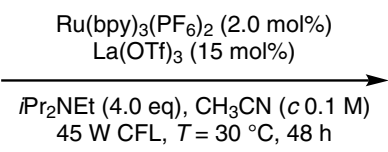

$\mathrm{Ru}(\mathrm{bpy})_{3}\left(\mathrm{PF}_{6}\right)_{2}(2.0 \mathrm{~mol} \%)$

$45 \mathrm{~W} \mathrm{CFL}, T=30^{\circ} \mathrm{C}, 48 \mathrm{~h}$

$1 a$<smiles>CC(C)[C@H]([C@H](O)c1ccc(F)cc1)N(O)Cc1ccccc1</smiles>

2a-1 (major)

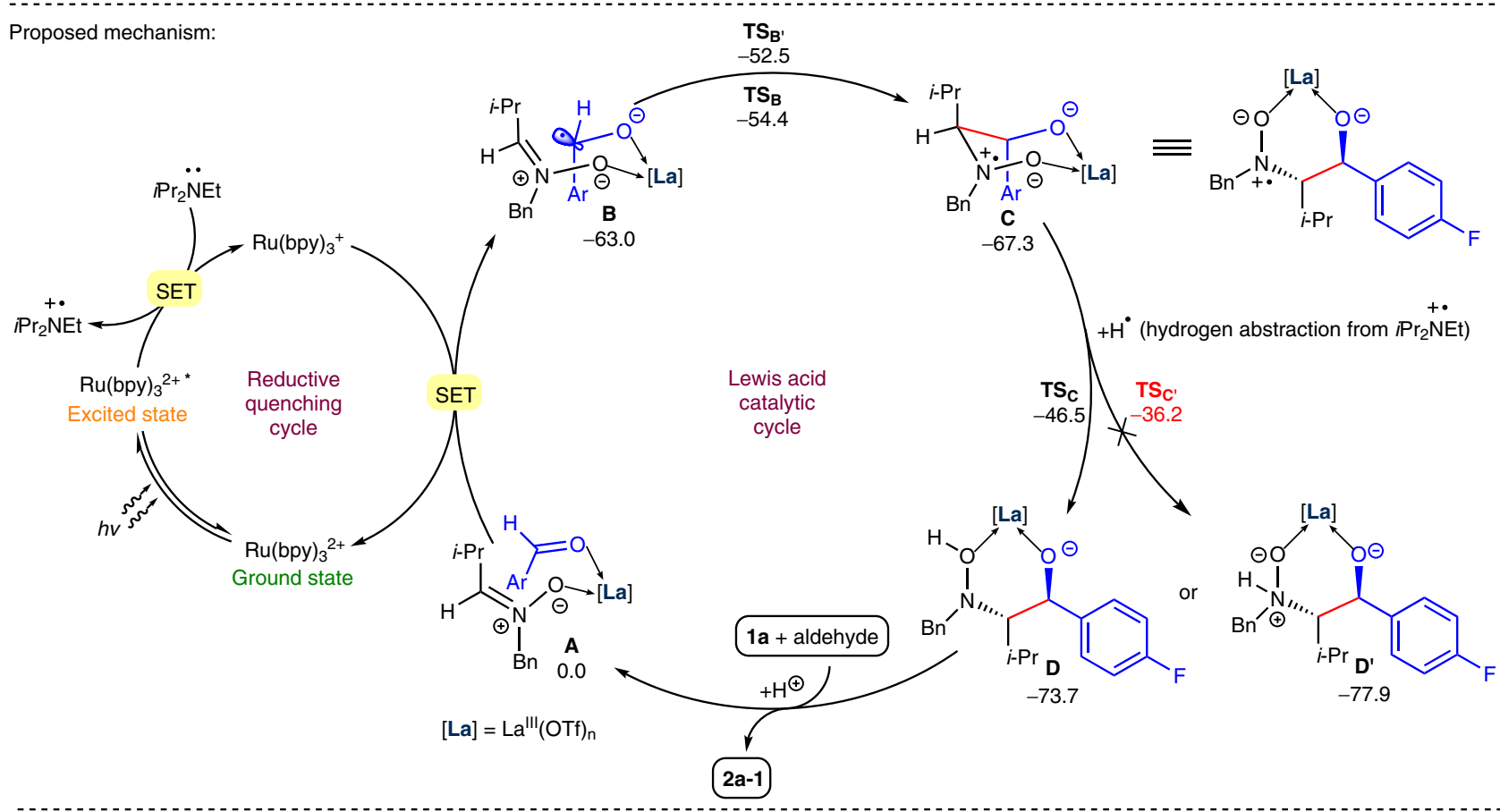

Plausible explanation of the stereoselectivity:

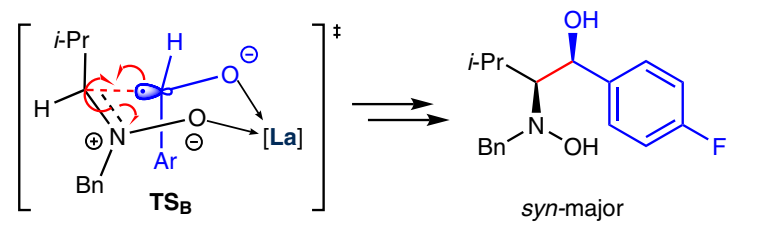

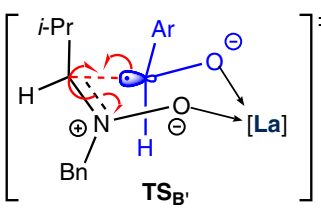

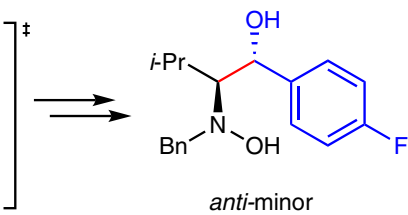
$\Delta G=1.9 \mathrm{kcal} / \mathrm{mo}$
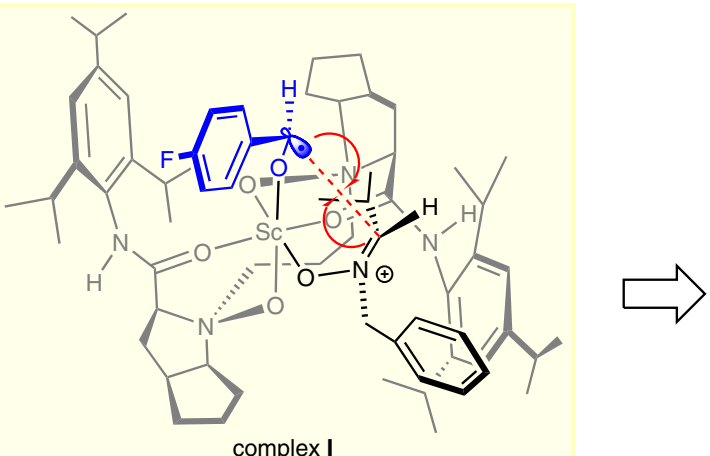<smiles>CC(C)C([Si])N(O)Cc1ccc(F)cc1</smiles>

Re face to $R e$ face attack

Fig. 3 Proposed mechanism of this photocatalytic enantioselective reductive cross-coupling reaction. Relative Gibbs free energies ( $\Delta G$ in $\mathrm{kcal}^{\mathrm{mol}}{ }^{-1}$ at 298 K) for key intermediates and transition states were computed at the SMD-B3LYP/DZP-level of theory

precursors, accounting well for the reaction specificity towards cross-coupling rather than homocoupling. On the basis of this mechanism, the diastereoselectivity of vicinal hydroxyamino alcohols, such as $\mathbf{2 a - 1}$, can be illustrated through comparing the energy of six-member ring transition state $\mathrm{TS}_{\mathrm{B}}$ with that of $\mathbf{T S}_{\mathbf{B}^{\prime}}$. The enantioselectivity is revealed by chiral scandium complex $\mathbf{I}^{36}$ involving a $R e$-to- $R e$-facial attack of the ketyl radical to nitrone 1a.
Concise synthesis of (+)-ephedrine and (-)-selegiline. With the dual aim of taking further insight into this reaction and demonstrating the utility of this enantioselective radical protocol, we undertook the synthesis of ephedrine and selegiline (Fig. 4). Following the modified method 1, asymmetric reductive coupling of nitrone $\mathbf{l n}$ with benzaldehyde gave product $\mathbf{2 n}$ as the major diastereomer $\left(85 \%\right.$ combined yield, $11 / 1 \mathrm{dr}$ ) with $94 \% \mathrm{ee}^{44}$. After the indium-mediated reduction ${ }^{39}$ of $2 \mathbf{n}$, vicinal amino alcohol $\mathbf{6 n}$ was obtained in $90 \%$ yield. The spectral data of $\mathbf{6}$ m matched those 


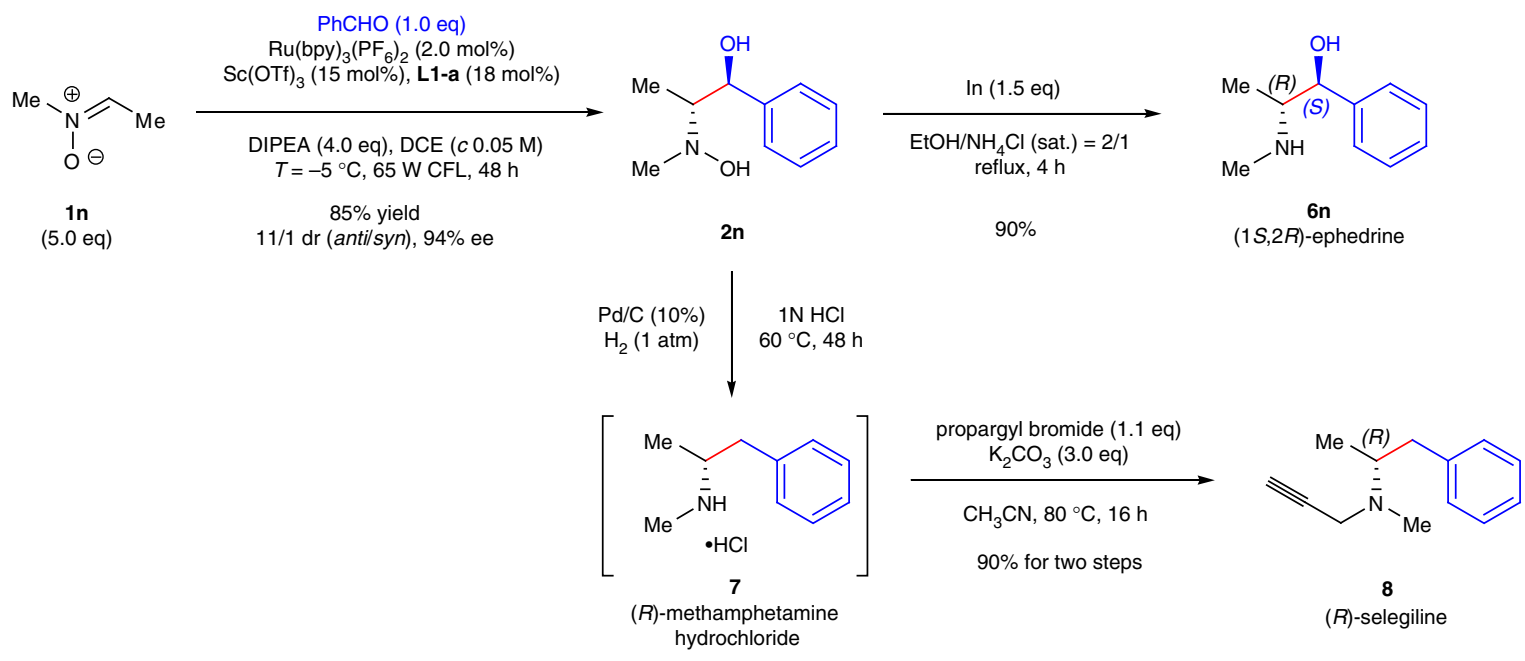

Fig. 4 Concise synthesis of (+)-ephedrine $\mathbf{6}$ n and (-)-selegiline 8. A concise two-step synthesis of (1S,2-R)-(+)-ephedrine $\mathbf{6 n}$ and an efficient three-step preparation of $(R)-(-)$-selegiline 8 have been achieved both with $70 \%$ overall yield and $94 \%$ ee

reported of ephedrine $\left[\mathbf{6} \mathbf{n} \bullet \mathbf{H C l}:[\alpha]_{\mathrm{D}}{ }^{20}=+30.6\left(c\right.\right.$ 2.0, $\left.\mathrm{H}_{2} \mathrm{O}\right)$; lit. ${ }^{45}$ for $(-)$-ephedrine $\left.\cdot \mathrm{HCl}:[\alpha]_{\mathrm{D}}{ }^{20}=-34.7\left(c 5.0, \mathrm{H}_{2} \mathrm{O}\right)\right]$. The optical rotation revealed that our synthetic vicinal amino alcohol $\mathbf{6 n}$ is $(1 S, 2 R)-(+)$-ephedrine. Although the relative stereochemistry of vicinal hydroxyamino alcohol $\mathbf{2 n}$ is different with the results listed in Table 2, this diastereoselectivity is supported by the DFT calculations that revealed the involvement of six-member ring transition state (see Supplementary Fig. 10). Furthermore, dehydroxylation of vicinal hydroxyamino alcohol $2 \mathbf{n}$ was carried out in an aqueous solution of $\mathrm{HCl}$ under a mild $\mathrm{Pd} / \mathrm{C}$ catalyzed hydrogenolysis condition to provide methamphetamine hydrochloride 7. $\mathrm{N}$-propargylation of crude 7 in acetonitrile with $\mathrm{K}_{2} \mathrm{CO}_{3}$ afforded (-)-selegiline (8) $\left[[\alpha]_{\mathrm{D}}{ }^{25}=-1.02\right.$ (c 1.0, EtOH); lit. ${ }^{46}$ for 8: $[\alpha]_{\mathrm{D}}{ }^{20}=-1.29$ (c $6.43, \mathrm{EtOH},>99 \%$ ee $\left.)\right]$ in $90 \%$ yield for two steps, which is a medicine used for the treatment of Parkinson's disease, depression, and senile dementia $^{47}$.

\section{Discussion}

In summary, we demonstrated a photocatalytic enantioselective reductive cross-coupling reaction of nitrones with aromatic aldehydes via the synergistic catalysis of Ru-photocatalyst and chiral $N, N^{\prime}$-dioxide ligand-coordinated rare earth ion. In this protocol, chiral Lewis acid represents an indispensable template for assembling to the key intermediate and triggers the asymmetric radical process to afford enantiopure vicinal hydroxyamino alcohols in moderate to excellent yields with high stereoselectivity. Taking advantage of this catalytic mechanism, unavoidable pinacol-type homocoupling side reactions in previous $\mathrm{SmI}_{2}$-mediated system were entirely inhibited. Notably, chiral $N, N^{\prime}$-dioxide ligands were used in a radical-mediated system to account for a high level of stereoselectivity. Furthermore, this reaction is operationally simple with a wide array of readily available substrates under mild condition, allowing for the stepeconomy synthesis of highly valuable enantiopure vicinal amino alcohols (e.g., $(1 S, 2 R)-(+)$-ephedrine) and amphetamine derivatives (e.g., $(R)-(-)$-selegiline) rivaling those of industrial biosynthetic procedures ${ }^{48}$. Based on a deep insight of this reaction, we believed that a foundation has been established for further research and application of these related reactions.

\section{Methods}

General. For ${ }^{1} \mathrm{H}$ and ${ }^{13} \mathrm{C}$ NMR spectra of compounds in this manuscript see Supplementary Methods. For details of the synthetic procedures and tables including detail experimental, see Supplementary Methods.
General procedure. An oven-dried $25 \mathrm{ml}$ Schlenk tube equipped with a magnetic stir bar was added nitrone $(0.30 \mathrm{mmol}, 1.0 \mathrm{eq}), \mathbf{L 1}-\mathbf{b}(42.4 \mathrm{mg}, 0.054 \mathrm{mmol}, 18 \mathrm{~mol}$ \%), $\mathrm{Ru}(\mathrm{bpy})_{3}\left(\mathrm{PF}_{6}\right)_{2}(5.2 \mathrm{mg}, 0.006 \mathrm{mmol}, 2.0 \mathrm{~mol} \%)$, and Sc(OTf $)_{3}(22.1 \mathrm{mg}, 0.045$ $\mathrm{mmol}, 15 \mathrm{~mol} \%)$ in the glove box. When the tube was sealed and removed from the glove box, DCE (or DCM) $(6.0 \mathrm{ml})$ was added, followed by the aldehyde $(0.45$ mmol, $1.5 \mathrm{eq})$, and TEEDA $(0.26 \mathrm{ml}, 1.2 \mathrm{mmol}, 4.0 \mathrm{eq})$. The tube was placed approximately $10 \mathrm{~cm}$ away from a $65 \mathrm{~W} \mathrm{CFL}$. After being stirred at $0{ }^{\circ} \mathrm{C}$ under an argon atmosphere for $48 \mathrm{~h}$, the reaction mixture was filtered through a thin pad of silica gel (100-200 mesh), washed with EtOAc, and concentrated under reduced pressure. The residue was purified by flash chromatography to afford desired vicinal hydroxyamino alcohols $\mathbf{2 a}$ to $\mathbf{2} \mathbf{j}$.

Modified method 1. An oven-dried $25 \mathrm{ml}$ Schlenk tube equipped with a magnetic stir bar was added nitrone $(0.30 \mathrm{mmol}, 1.0 \mathrm{eq}), \mathbf{L 1}-\mathbf{a}(37.9 \mathrm{mg}, 0.054 \mathrm{mmol}, 18 \mathrm{~mol}$ $\%), \mathrm{Ru}(\mathrm{bpy})_{3}\left(\mathrm{PF}_{6}\right)_{2}(5.2 \mathrm{mg}, 0.006 \mathrm{mmol}, 2.0 \mathrm{~mol} \%)$, and $\mathrm{Sc}(\mathrm{OTf})_{3}(22.1 \mathrm{mg}, 0.045$ $\mathrm{mmol}, 15 \mathrm{~mol} \%)$ in the glove box. When the tube was sealed and removed from the glove box, DCM $(6.0 \mathrm{ml})$ was added, followed by the aldehyde $(0.45 \mathrm{mmol}, 1.5 \mathrm{eq})$, and DIPEA $(0.21 \mathrm{ml}, 1.2 \mathrm{mmol}, 4.0 \mathrm{eq})$. The tube was placed approximately $10 \mathrm{~cm}$ away from a $65 \mathrm{~W}$ CFL and the reaction mixture was stirred at $-5^{\circ} \mathrm{C}$ under an argon atmosphere for $48 \mathrm{~h}$ to afford desired products $2 \mathbf{k}$ to $2 \mathbf{m}$.

Modified method 2. An oven-dried $25 \mathrm{ml}$ Schlenk tube equipped with a magnetic stir bar was added nitrone $(0.30 \mathrm{mmol}, 1.0 \mathrm{eq}), \mathbf{L 2}-\mathbf{b}(32.4 \mathrm{mg}, 0.09 \mathrm{mmol}$, $30 \mathrm{~mol} \%), \mathrm{Ru}(\mathrm{bpy})_{3}\left(\mathrm{PF}_{6}\right)_{2}(5.2 \mathrm{mg}, 0.006 \mathrm{mmol}, 2.0 \mathrm{~mol} \%)$, and $\mathrm{La}(\mathrm{OTf})_{3}(26.4 \mathrm{mg}$, $0.045 \mathrm{mmol}, 15 \mathrm{~mol} \%)$ in the glove box. When the tube was sealed and removed from the glove box, $\mathrm{CH}_{3} \mathrm{CN}(6.0 \mathrm{ml})$ was added, followed by the aldehyde $(0.45 \mathrm{mmol}, 1.5 \mathrm{eq})$ and TEEDA $(0.26 \mathrm{ml}, 1.2 \mathrm{mmol}, 4.0 \mathrm{eq})$. The tube was placed approximately $10 \mathrm{~cm}$ away from a $65 \mathrm{~W}$ CFL and the reaction mixture was stirred at $-10^{\circ} \mathrm{C}$ under an argon atmosphere for $72 \mathrm{~h}$ to afford desired products $2 \mathrm{k}$ to $\mathbf{2 m}$.

Data availability. The crystallographic data have been deposited at the Cambridge Crystallographic Data Centre (CCDC) as CCDC $1537335(\mathbf{6 a}-\mathbf{9} \cdot \mathbf{H C l}), 1537337$ $(6 \mathbf{b}-3 \bullet \mathbf{H C l})$, and $1537338(\mathbf{6 k - 3 \bullet} \mathrm{HCl})$, and can be obtained free of charge from www.ccdc.cam.ac.uk/structures. Any further relevant data are available from the authors upon reasonable request.

Received: 20 July 2017 Accepted: 13 December 2017

Published online: 29 January 2018

\section{References}

1. Reetz, M. T. Synthesis and diastereoselective reactions of $N, N$-dibenzylamino aldehydes and related compounds. Chem. Rev. 99, 1121-1162 (1999).

2. Bergmeier, S. C. The synthesis of vicinal amino alcohols. Tetrahedron $\mathbf{5 6}$, 2561-2576 (2000).

3. Klingler, F. D. Asymmetric hydrogenation of prochiral amino ketones to amino alcohols for pharmaceutical use. Acc. Chem. Res. 40, 1367-1376 (2007).

4. Métro, T.-X., Duthion, B., Pardo, D. G. \& Cossy, J. Rearrangement of $\beta$-amino alcohols via aziridiniums: a review. Chem. Soc. Rev. 39, 89-102 (2010). 
5. Weng, C., Zhang, H., Xiong, X., Lu, X. \& Zhou, Y. Evolution of epoxides to synthesize $\beta$-amino alcohols. Asian J. Chem. 26, 3761-3768 (2014).

6. Li, G., Chang, H.-T. \& Sharpless, K. B. Catalytic asymmetric aminohydroxylation (AA) of olefins. Angew. Chem. Int. Ed. Engl. 35, 451-454 (1996).

7. Donohoe, T. J., Callens, C. K. A., Flores, A., Lacy, A. R. \& Rathi, A. H. Recent developments in methodology for the direct oxyamination of olefins. Chem. Eur. J. 17, 58-76 (2011).

8. Sasai, H. in Comprehensive Organic Synthesis II (eds. Knochel P. \& Molander G. A.). Ch 2.13 (Elsevier, Amsterdam, 2014).

9. Burchak, O. N. \& Py, S. Reductive cross-coupling reactions (RCCR) between $\mathrm{C}=\mathrm{N}$ and $\mathrm{C}=\mathrm{O}$ for $\beta$-amino alcohol synthesis. Tetrahedron $65,7333-7356$ (2009).

10. Zhong, Y.-W. et al. A highly efficient and direct approach for synthesis of enantiopure $\beta$-amino alcohols by reductive cross-coupling of chiral $N$-tertbutanesulfinyl imines with aldehydes. J. Am. Chem. Soc. 127, 11956-11957 (2005).

11. Lin, G.-Q., Xu, M.-H., Zhong, Y.-W. \& Sun, X.-W. An advance on exploring Ntert-butanesulfinyl imines in asymmetric synthesis of chiral amines. Acc. Chem. Res. 41, 831-840 (2008).

12. Masson, G., Py, S. \& Vallée, Y. Samarium diiodide-induced reductive crosscoupling of nitrones with aldehydes and ketones. Angew. Chem. Int. Ed. 41, 1772-1775 (2002).

13. Burchak, O. N., Philouze, C., Chavant, P. Y. \& Py, S. A direct and versatile access to $\alpha, \alpha$-disubstituted 2-pyrrolidinylmethanols by $\mathrm{SmI}_{2}$-mediated reductive coupling. Org. Lett. 10, 3021-3023 (2008).

14. Wu, S.-F., Zheng, X., Ruan, Y.-P. \& Huang, P.-Q. A new approach to 3 hydroxyprolinol derivatives by samarium diiodide-mediated reductive coupling of chiral nitrone with carbonyl compounds. Org. Biomol. Chem. 7, 2967-2975 (2009).

15. Wu, S.-F., Ruan, Y.-P., Zheng, X. \& Huang, P.-Q. Samarium diiodide-mediated reductive couplings of chiral nitrones with aldehydes/ketones and acyl chlorides. Tetrahedron 66, 1653-1660 (2010).

16. Riber, D., Hazell, R. \& Skrydstrup, T. Studies on the SmI2-promoted pinacoltype cyclization: synthesis of the hexahydroazepine ring of balanol. J. Org. Chem. 65, 5382-5390 (2000).

17. Narayanam, J. M. R. \& Stephenson, C. R. J. Visible light photoredox catalysis: applications in organic synthesis. Chem. Soc. Rev. 40, 102-113 (2011).

18. Prier, C. K., Rankic, D. A. \& MacMillan, D. W. C. Visible light photoredox catalysis with transition metal complexes: applications in organic synthesis. Chem. Rev. 113, 5322-5363 (2013).

19. Hopkinson, M. N., Sahoo, B., Li, J.-L. \& Glorius, F. Dual catalysis sees the light: combining photoredox with organo-, acid, and transition-metal catalysis. Chem. Eur. J. 20, 3874-3886 (2014).

20. Beatty, J. W. \& Stephenson, C. R. J. Amine functionalization via oxidative photoredox catalysis: methodology development and complex molecule synthesis. Acc. Chem. Res. 48, 1474-1484 (2015).

21. Skubi, K. L., Blum, T. R. \& Yoon, T. P. Dual catalysis strategies in photochemical synthesis. Chem. Rev. 116, 10035-10074 (2016).

22. Chen, J.-R., Hu, X.-Q., Lu, L.-Q. \& Xiao, W.-J. Visible light photoredoxcontrolled reactions of $\mathrm{N}$-radicals and radical ions. Chem. Soc. Rev. 45, 2044-2056 (2016).

23. Rono, L. J., Yayla, H. G., Wang, D. Y., Armstrong, M. F. \& Knowles, R. R. Enantioselective photoredox catalysis enabled by proton-coupled electron transfer: development of an asymmetric aza-pinacol cyclization. J. Am. Chem. Soc. 135, 17735-17738 (2013).

24. Ma, J., Harms, K. \& Meggers, E. Enantioselective rhodium/ruthenium photoredox catalysis en route to chiral 1,2-aminoalcohols. Chem. Commun. 52, 10183-10186 (2016).

25. Wang, C. et al. Asymmetric radical-radical cross-coupling through visible-lightactivated iridium catalysis. Angew. Chem. Int. Ed. 55, 685-688 (2016).

26. Fava, E., Millet, A., Nakajima, M., Loescher, S. \& Rueping, M. Reductive umpolung of carbonyl derivatives with visible-light photoredox catalysis: direct access to vicinal diamines and amino alcohols via $\alpha$-amino radicals and ketyl radicals. Angew. Chem. Int. Ed. 55, 6776-6779 (2016)

27. Hager, D. \& MacMillan, D. W. C. Activation of C-H bonds via the merger of photoredox and organocatalysis: a coupling of benzylic ethers with Schiff bases. J. Am. Chem. Soc. 136, 16986-16989 (2014).

28. Musacchio, A. J. et al. Catalytic intermolecular hydroaminations of unactivated olefins with secondary alkyl amines. Science 355, 727-730 (2017).

29. Huo, H. et al. Asymmetric photoredox transition-metal catalysis activated by visible light. Nature 515, 100-103 (2014).

30. Lin, C.-W., Hong, B.-C., Chang, W.-C. \& Lee, G.-H. A new approach to nitrones through cascade reaction of nitro compounds enabled by visible light photoredox catalysis. Org. Lett. 17, 2314-2317 (2015).

31. Mikami, K., Terada, M. \& Matsuzawa, H. "Asymmetric" catalysis by lanthanide complexes. Angew. Chem. Int. Ed. 41, 3554-3571 (2002).
32. Aspinall, H. C. Chiral lanthanide complexes: coordination chemistry and applications. Chem. Rev. 102, 1807-1850 (2002).

33. Lam, Y.-H., Grayson, M. N., Holland, M. C., Simon, A. \& Houk, K. N. Theory and modeling of asymmetric catalytic reactions. Acc. Chem. Res. 49, 750-762 (2016).

34. Zimmerman, H. E. \& Traxler, M. D. The stereochemistry of the Ivanov and Reformatsky reactions. I. J. Am. Chem. Soc. 79, 1920-1923 (1957).

35. Yu, Z., Liu, X., Dong, Z., Xie, M. \& Feng, X. An $N, N^{\prime}$-dioxide/In $(\mathrm{OTf})_{3}$ catalyst for the asymmetric hetero-Diels-Alder reaction between Danishefsky's dienes and aldehydes: application in the total synthesis of triketide. Angew. Chem. Int. Ed. 47, 1308-1311 (2008).

36. Liu, X., Lin, L. \& Feng, X. Chiral $N, N^{\prime}$-dioxides: new ligands and organocatalysts for catalytic asymmetric reactions. Acc. Chem. Res. 44, 574-587 (2011).

37. Yoon, T. P. \& Jacobsen, E. N. Privileged chiral catalysts. Science 299, 1691-1693 (2003).

38. Espelt, L. R., McPherson, I. S., Wiensch, E. M. \& Yoon, T. P. Enantioselective conjugate additions of $\alpha$-amino radicals via cooperative photoredox and Lewis acid catalysis. J. Am. Chem. Soc. 137, 2452-2455 (2015).

39. Cicchi, S., Bonanni, M., Cardona, F., Revuelta, J. \& Goti, A. Indiummediated reduction of hydroxylamines to amines. Org. Lett. 5, 1773-1776 (2003).

40. Bowry, V. W., Lusztyk, J. \& Ingold, K. U. Evidence for reversible ring-opening of the $\alpha$-cyclopropylbenzyl radical. J. Chem. Soc. Chem. Commun. 26, 923-925 (1990).

41. Xu, J., Samsuri, N. B. \& Duong, H. A. Nickel-catalysed cyclopropanation of electron-deficient alkenes with diiodomethane and diethylzinc. Chem. Commun. 52, 3372-3375 (2016).

42. Juris, A., Balzani, V., Belser, P. \& von Zelewsky, A. Characterization of the excited state properties of some new photosensitizers of the ruthenium (polypyridine) family. Helv. Chim. Acta 64, 2175-2182 (1981).

43. Kalyanasundaram, K. Photophysics, photochemistry and solar energy conversion with tris(bipyridyl)ruthenium(II) and its analogues. Coord. Chem. Rev. 46, 159-244 (1982).

44. O'Neil, I. A. et al. The synthesis and structure of chiral enamine $N$-oxides. Chem. Commun. 50, 7336-7339 (2014).

45. Raffa, R. J., Stern, M. J. \& Malspeis, L. Thermometric titration determination of $\Delta \mathrm{H}^{\circ}, \Delta \mathrm{G}^{\circ}$, and $\Delta \mathrm{S}^{\circ}$ of dissociation of ephedrinium and pseudoephedrinium ions. Anal. Chem. 40, 70-77 (1968)

46. Bornholdt, J., Felding, J., Clausen, R. P. \& Kristensen, J. L. Ring opening of pymisyl-protected aziridines with organocuprates. Chem. Eur. J. 16, 12474-12480 (2010).

47. Riederer, P., Lachenmayer, L. \& Laux, G. Clinical applications of MAOinhibitors. Curr. Med. Chem. 11, 2033-2043 (2004).

48. Panke, S. \& Wubbolts, M. Advances in biocatalytic synthesis of pharmaceutical intermediates. Curr. Opin. Chem. Biol. 9, 188-194 (2005).

\section{Acknowledgements}

We are grateful to the NSF of China $(21472157,21672175,21332007,21273177$, and 91545105), the Fundamental Research Funds for the Central Universities (No. 20720160048), the NFFTBS (No. J1310024), and the Program for Changjiang Scholars and Innovative Research Team in University (PCSIRT) for financial support. We also thank Dr. Xiao-Yu Cao of XMU for kind and helpful discussions on this paper, and $\mathrm{Mr}$. Zi-Ang Nan at iChEM for recrystallization for X-ray quality crystals, X-ray crystallographic analysis with refinement, and valuable discussions.

\section{Author contributions}

C.-X.Y. discovered the reaction. C.-X.Y., H.-H.L., and J.-T.C. performed the experiments and analyzed the data. Y.-P.R. and T.-T.Z. performed the chiral HPLC analysis. X.L. and Y.Y.M. performed computational studies on the mechanism. X.Z. and C.-X.Y. designed the project and wrote the manuscript. Feng's chiral ligand was suggested by P.-Q.H. X.Z., X.L., and P.-Q.H. directed the project and polished the manuscript. All of the authors discussed the results and commented on the manuscript.

\section{Additional information}

Supplementary Information accompanies this paper at https://doi.org/10.1038/s41467017-02698-4.

Competing interests: The authors declare no competing financial interests.

Reprints and permission information is available online at http://npg.nature.com/ reprintsandpermissions/

Publisher's note: Springer Nature remains neutral with regard to jurisdictional claims in published maps and institutional affiliations. 
(c) (i) Open Access This article is licensed under a Creative Commons Attribution 4.0 International License, which permits use, sharing, adaptation, distribution and reproduction in any medium or format, as long as you give appropriate credit to the original author(s) and the source, provide a link to the Creative Commons license, and indicate if changes were made. The images or other third party material in this article are included in the article's Creative Commons license, unless indicated otherwise in a credit line to the material. If material is not included in the article's Creative Commons license and your intended use is not permitted by statutory regulation or exceeds the permitted use, you will need to obtain permission directly from the copyright holder. To view a copy of this license, visit http://creativecommons.org/ licenses/by/4.0/.

(C) The Author(s) 2017 